\title{
Analysis of Urban Greenway Planning Strategies by The Orientation of Healthy City
}

\author{
Qiutong Chen ${ }^{l}$,Ziliu Qin ${ }^{1}$, Xiang $\mathrm{Ji}^{l}$, Shuang Ming ${ }^{l}$, and Qin Du ${ }^{l, *}$ \\ ${ }^{1}$ College of Tourism \& Landscape Architecture, Guilin University of Technology, Guilin 541006, China
}

\begin{abstract}
In the first half-year of 2020, the COVID-19 (Coronavirus disease 2019) outbreak spread rapidly around the world. Under the impact of the crisis of Public Health Emergency, the development of healthy cities and people's lives must be experienced a tough test, and it will trigger the thinking and practice of urban construction as well. Taking the construction of Nanning greenway system as an example, and trying to discuss the route and the significance of healthy cities construction by greenway supporting, we came up with suggestions for greenway construction by the orientation of the concept healthy city.
\end{abstract}

\section{Introduction}

In recent years, public health issues caused by the urban environment have gradually gained more attention, challenging the prevention and control capabilities of the urban public health crisis. More than a few hundred years ago, countries in Europe, America, and other regions have suffered from severe public health crises. Dengue fever in the 18th century and the Cholera epidemic that swept the world in the 19th century threatened human health and social stability. In early 2020, the COVID-19 outbreak spread rapidly, raging in many provinces and cities in China. At the beginning of March, other parts of Asia, Europe, and America were also affected by the virus. During the period of virus-fighting, cities in our countries can organize prevention and control measures quickly and orderly, such as community mutual assistance and selfhelp, which played a role that cannot be ignored during the disaster. This international public health event has also renewed our reflections on urban planning and spatial design strategies for resolving the crisis of Public Health Emergency. The planning and design for the urban green space in the future should pay attention to the prevention, response and recovery of such public emergencies, and improve the overall prevention and control capabilities of the city. Although provinces and cities around the country have made healthy city construction an important goal of urban planning, the current practice of healthy city construction in China is still unable to penetrate into urban environmental construction or spatial planning. A large number of measures are limited to the satisfaction of basic urban sanitation conditions ${ }^{[1]}$.

Greenway as a linear green open space, in the form of the multiple scale and functional characteristics, be meet the demand of urban and rural ecological construction and residents daily recreation important spatial carrier, its integrity and connectivity advantages to solve the life of infrastructure and public leisure space accessibility and fairness problem solving strategy provides, to promote the construction of healthy city. In recent years, many urban planning scholars have introduced foreign health impact assessment methods to evaluate the urban construction projects in China, but little discussion has been made on the significance of greenway construction for healthy city and the optimal path of greenway construction in China under the guidance of healthy city. Based on the requirements of healthy city construction, this paper analyzes the following two contents: 1) the path and significance of urban greenway to promote the construction of healthy city;2) take nanning city as an example to put forward the strategy of urban greenway construction under the guidance of healthy city.

\section{Healthy Cities and Greenways}

\subsection{The Concept of Healthy City}

In the 1980s, the World Health Organization (WHO) took the initiative to build healthy cities as a global action strategy in the face of the human health challenges caused by the deterioration of the urban environment. The healthy city should be a city that constantly develops, develops the natural and social environment, and expands social resources so that people can support each other in enjoying life and giving full play to their potential.

\subsection{Healthy City Construction Routes}

The core of healthy city construction is to combine with 'healthy people, a healthy environment, and healthy society' organically. Health is a comprehensive problem of multi-disciplinary related influence, and health, life behavior and healthy environment are mutually influential relationships (Figure 1).

\footnotetext{
* Corresponding author: 80168391@qq.com
} 
Studies have shown that social factors are the most prominent of the factors that affect human health. Infrastructure supply, social interaction patterns, surrounding land used, economic and cultural conditions, etc. will have a positive or negative potential impact on residents. Healthy cities lead the healthy lifestyle of urban residents indirectly through the layout adjustment, spatial planning, and the improvement of landscape quality, it takes social equity and the overall well-being of the city into account to achieve human physical, mental and social health at the same time.

The orientation of the concept of a healthy city pays more attention to physical health services, psychological health regulation, and social health construction. Physiological health services attach importance to alleviating the health and immunity problems of urban residents. Various sub-health problems need to be improved, such as obesity, low immunity, and chronic diseases. In addition, we must pay attention to the tolerance of vulnerable groups.

Mental health mainly focuses on whether the open space design can regulate the emotional stress of users, ease the cure of mental illness, and enhance health awareness $^{[2]}$. While social health services focus on building spaces for public social activities, ensuring fairness and equality in service facilities, enhancing a sense of security, reducing violent crime, and enhancing urban cohesion and belonging.

According to the demand for green space functions in healthy cities, the urban greenway planning and design route are proposed to improve the long-term resistance of cities to health problems, the timely response to emergent crises, and the resilience after crises ${ }^{[3]}$. So that we can optimize the planning of system route selection, design, and management methods ${ }^{[4]}$.

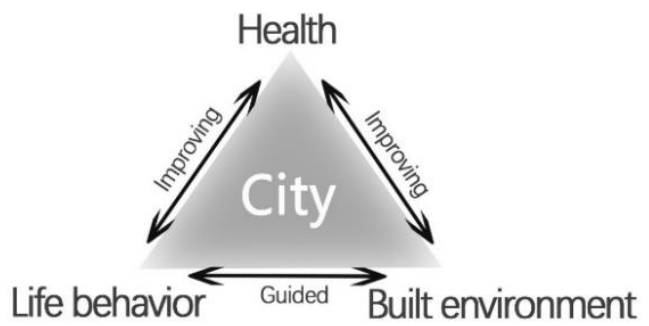

Fig. 1. Healthy City Construction Relationship

\subsection{The Importance of Urban Greenways to The Construction of Healthy Cities}

Greenway is a kind of green infrastructure with comprehensive functions of ecology, leisure and recreation, natural and historical and cultural display, disaster reduction, and risk avoidance, and many studies have shown that the greenway has significant health benefits, such as alleviating sub-health problems. Bringing multi-dimensional health connotations into the planning and design of urban greenways is of great significance for the greenway's ability to support public health (Table 1).
Table 1. Healthy city content.

\begin{tabular}{|c|c|}
\hline Healthy city & status quo and needs \\
\hline \multirow{5}{*}{ physical health } & Sub-health, \\
& Chronic disease, \\
& Handicapped person, \\
& Hypo immunity, \\
& Allergen susceptibility, \\
& An epidemic, \\
etc.
\end{tabular}

\subsubsection{Enhance long-term resistance of urban health}

The changes in the lifestyles of residents in modern society have greatly reduced the need for urban residents to go out. Some people spend their free time on outdoor exercise. The gradual increase of urban residents' attention to sports greenways is an opportunity to improve health problems. Faced with the outbreak of public health crisis, a healthy body can greatly enhance the residents' ability to resist crisis.

The green road is not only a support for healthy physical activities such as cycling, walking and lowcarbon transportation, but also a linear open spaces and nodes connecting with natural landscapes, which can be a place for tourism to rest and physical exercise, being conducive to residents to strengthen physical exercise and reduce disease. The strong connectivity of walking between regions and communities, reflecting the inclusive of elderly, children and people with reduced mobility, and strengthening the communication of neighbors. The continuous green space has a certain effect on the regulation of emotions and the relief of stress, which helps to improve the problems of patients with sub-health and chronic diseases. The greenway space is wedged between urban communities and streets to regulate the local microclimate, purify the air, and reduce the occurrence of respiratory diseases. Generally, green aisles are connected to urban natural scenic areas, and become the best place for outdoor science learning, to show the characteristics of the local style, to enhance the awareness of urban residents on the protection of urban mountain water resources and regional culture, to enhance the city's healthy and harmonious atmosphere and safety sense, reduce the crime rate.

\subsubsection{Improve urban health and timely response}

Unexpected crises such as natural disasters or public health crises are difficult to predict. When cities and 
residents face crises, timely response measures can reduce the damage of disasters to the socio-economic environment and reduce residents' health risks. From the perspective of greenway construction, how to use its benefits to improve the ability of cities and residents to respond to crises should be included in the content of greenway planning and design.

The greenway is an open space, which can provide places for disaster avoidance, temporary evacuation, and settlement; the vegetation isolation zone along the greenway has a certain blocking effect on pollutants, and the designer can benefit from the harmful substances in the air through reasonable plant configuration and absorption. By adjusting the width and dimension of the open space guide greenway users to maintain a safe and hygienic social distance, reduce the cross-infection of bacteria. Greenways in a modern society mostly use smart management systems such as real-time monitoring of the number of users, congestion, and safe route guidance. When an emergency occurs, these facilities can be used to avoid confusion by a reasonable channel.

\subsubsection{Improving the resilience of cities after disasters}

The outbreak of urban emergency will inevitably have a negative impact on the social atmosphere and residents' sentiment. When the infectious disease outbreak, scenic spots, gymnasiums, and various exhibition halls will be closed to avoid gathering activities. The problem of how to alleviate the pressure and the tense of urban atmosphere should be resolved first. Studies have shown that people perform low-intensity activities in a green landscape space can increase positive feelings by $60 \%$ and selfesteem by $70 \%$. Urban greenways can play the value of natural healing and provide a place to relieve bad moods ${ }^{[5]}$.

\section{Overview of The Construction of Greenway System in Nanning}

In 2013, Nanning City Greenway System Planning plans to build a total length of $437.33 \mathrm{~km}$ of greenways, including 2 municipal-level greenways totaling $148.61 \mathrm{~km}$, 12 central city-level greenways totaling $125.93 \mathrm{~km}$, and 19 group-level greenways A total of $162.80 \mathrm{~km}$. Specifically, Qingxiu Mountain's core scenic spots, Yongjiang and other cycling greenways and riverside greenways are well known and used by citizens, but there are still many problems that restrict Nanning's greenways from exerting their health benefits.

\subsection{Weak Perception of Greenway Facilities among Residents}

Apart from the Qingxiu Mountain Scenic Area cycling road and the Yongjiang Customs Corridor Green Road that are welcomed by more users due to its superior natural landscape advantages and perfect facility service system. There are most of the greenways that have been built are not well known to the citizens, such as the outer ring greenway in the center of the city, and the ecological leisure corridors around the remaining water systems in the center. On the one hand, the poor connection between the residents' residence and the entrance of the greenway is restricted to the use of the greenway and the green space facilities that far from the residence. On the other hand, the single design form reduces the attractiveness of the greenway and lacks attention to users of different ages; the management and operation system is not perfect after the completion of the greenway, and the citizens know very little about the greenway-related information. Overall, it failed to attract and orient citizens to use greenways to participate in healthy exercise activities.

\subsection{Lack of Security Management for Green Road}

There are large loopholes of management and maintenance in the greenway on the outer edge of downtown Nanning, due to the low daily flow, the simple environmental facilities, and the tourist's lack of security awareness. As a result, there are certainly hidden security risks as well. The selection of the green roads in the central area is mostly along the waterline, and the warning signs are not obvious and not completed enough, the first aid measures are not perfect enough are all important reasons for the security incident.

\subsection{The Imperfect Design of Greenway Health Protection}

The daily flow of green roads in urban centers and scenic areas is large. Tourists at rest stations and scenic spots are easy to gather. At this stage, most green road facilities are designed with poor consideration of sanitation and epidemic prevention measures. So the greenway health protection measures need to be improved urgently.

\subsection{Insufficient sustainability of greenway vitality}

At present, the green road landscape function experience in Nanning is similar, and tourists are prone to burnout. Greenway entertainment facilities only meet the daily use of residents in the surrounding elderly and children's age groups, while younger groups tend to join in clustered and distinctive sports activities. The waters along the greenway and surrounding scenic spots are not fully utilized as venues for events, and there is a low level of greenway use.

\section{Suggestions of Nanning Greenway Planning Optimization Oriented by Healthy City}

\subsection{Enhancing Recognizability and Attractiveness of Greenways}

Relying on the synchronous implementation of Nanning's urban style construction, strengthening the theme image of greenway planning, exploring regional culture, the urban style will be displayed, urban mountain water 
resources and the humanistic advantages will be got more effective attention and systematic protection, the recognizability of greenway routes can be improved at the same time. The construction of Nanning 's greenway system can pay more attention to some urban recession areas, such as the historical old city area to be renovated, ecologically polluted land to be restored, and industrial wasteland. In this way to accelerate urban renewal and increase the short-distance connection of communitylevel green trails or walking trails at the same time. Children and the elderly are more likely to use the greenway than the middle-aged group. Due to the limitation of physical quality and behavioral ability, the distance to the greenway and the demand for its use characteristics are more sensitive. Therefore, more attention should be paid to the increase of corresponding facilities, the design of barrier-free use, health knowledge, and the publicity and guidance of health awareness.

\subsection{Greenway Smart Management Operation System Implementation}

The city greenway with smart, efficient, safe and orderly, driving vitality characters, leading the healthy development can meet the trend of requirements for the construction of modern healthy cities. From the perspective of managers, Greenway should pay attention to the development of special management measures for the purpose of preventing and controlling emergent safety and health incidents. The big data center platform can be used for real-time monitoring, one-key alarms, and crowd evacuation control programs. Quickly make targeted responses and linkages to improve management and reduce losses. From the user's point of view, the mobile terminal basic software platform, cloud platform system services, etc. are used to provide a full range of comprehensive services, including tour route selection, safe passage recommendations, real-time preview of facility locations and passage congestion, Route guidance, and popular sports knowledge, etc. to meet the diverse needs of citizens and tourists.

\subsection{Improving Greenway Sanitation Prevention and Control Capabilities}

The design of the micro-green space along the greenway should not only facilitate air circulation but also consider the blocking of viral pollutants, selecting plant species resistant to dust pollutants, and supporting the vegetation hierarchy of air circulation. The scale and ventilation of public gathering activity spaces and the design of crowd evacuation guidance are particularly important. The establishment of vegetation isolation belts on the side of areas with high population concentration reduces the risk of virus transmission. For open spaces with high activity density, you can also adjust the distance and scale of the facility according to the spread distance, suspension time, etc. of the crowd talking, sneezing, coughing, etc. to avoid cross-infection, and set up temporary epidemic prevention devices and seasonal epidemic prevention systems to enhance the overall Hygiene prevention and control capabilities.

\subsection{Sustainable Construction of Greenway Vitality}

After the public health crisis, use the waterfront greenway and the city greenway to carry out various sports competitions, such as marathons, water sports, etc., organize community activities in the community green space node space, in order to maintain the vitality of the greenway and encourage people to get close to nature. Thematic greenway can help to improve the utilization rate. Depending on the cultural advantages along the greenway, it enriches the landscape content, relieve stress and anxiety, prevent discrimination in epidemic areas, eliminate urban loneliness, maintain the self-confidence and sense of belonging of urban residents, and at the same time increase the value of land along the route and promote the rapid recovery of the tourism and leisure economy ${ }^{[6]}$.

\section{Conclusion}

Based on the concept of healthy city construction and the goal of building a healthy urban environment, this paper discusses the opportunities and challenges of greenway system planning and design to improve the urban socialecological environment and residents' health problems, believing that greenway optimization construction can make the city have a certain resistance, coping ability and resilience to emergent health events and long-term healthy development, and put forward the optimization strategy of urban greenway planning and design. After achieved certain positive results in our country, it is very necessary to introspect to and optimize urban green space planning and design. Through the guidance and adjustment of the built environment to the lives of urban residents, the overall crisis immunity of the city will be improved to prevent future problems, is one of the important means of urban healthy and sustainable development. Urban greenway construction should pay more attention to the study of differentiated needs in the future, and pay attention to the needs of users of different ages, and support the health impact assessment of greenway design to maximize the benefits of urban greenways.

\section{Acknowledgement}

We thank the Natural Science Foundation of Guangxi Province(Nos.2018GXNSFAA050068, 2018JJB130217) and the Special Project of the Ministry of Science and Technology (2019YFC0507500-3) for funding support.

\section{References}

1. Y.C.Wang, J.K.Shen, Z.W.Peng, W.N.Xiang. Service optimization of green infrastructure 
ecosystem for urban growth [J]. Chinese landscape architecture, 2008,34 (10) : 45-49.

2. J.Xiao, S.P.Li, S.Liang. Building and strategy of resilient urban planning model for health [J]. Planner, 2020,36 (06) : 61-64.

3. Y.Xu, Y.P.Zhang, W.N.Wang, J.L.Su. Study on the planning characteristics and use factors of sports parks from the perspective of healthy city [J]. Chinese landscape architecture, 2008,34 (05) : 71-75.

4. L.Wang, S.W.Liao, X.J.Zhao. Analysis on the path and elements of healthy city planning [J]. International urban planning, 2016,31 (04) : 4-9.

5. Y.Wang. Community planning under the guidance of healthy city [J]. Planner, 2015,31 (10) : 101-105.

6. F.Z.Li, Y.L.Zhang, X.Li. Discussion on the construction method of urban greenway system based on the protection of landscape resources -- a case study of the greenway planning in lengshuijiang city [J]. Chinese landscape architecture, 2014,30 (08) : 51-54. 